\title{
The putative tumor suppressor gene GLTSCR2 induces PTEN-modulated cell death
}

\author{
J-H Yim ${ }^{1,2}$, Y-J Kim ${ }^{1,2}$, J-H Ko ${ }^{1,2}$, Y-E Cho ${ }^{1,2}$, S-M Kim ${ }^{1,2}$, J-Y Kim ${ }^{1,2}$, S Lee ${ }^{1,2}$ and J-H Park, ${ }^{\star, 1,2}$
}

Glioma tumor suppressor candidate region gene 2 (GLTSCR2/PICT-1) is localized within the well-known 1.4-Mb tumor suppressive region of chromosome 19q, which is frequently altered in various human tumors, including diffuse gliomas. Aside from its localization on the chromosome, several lines of evidence, such as PTEN phosphorylation, support that GLTSCR2 partakes in the suppression of tumor growth and development. However, much remains unknown about the molecular mechanisms of the tumor suppressive activity of GLTSCR2. The purpose of this study was to investigate the molecular mechanisms of GLTSCR2 in cell death pathways in association with its binding partner PTEN. In this work, we show that GLTSCR2 is a nucleus-localized protein with a discrete globular expression pattern. In addition to phosphorylating PTEN, GLTSCR2 induces caspase-independent PTEN-modulated apoptotic cell death when overexpressed. However, the cytotoxic activity of GLTSCR2 is independent of its ability to phosphorylate PTEN, suggesting that the GLTSCR2-induced cell death pathway is divergent from PTEN-induced death pathways. Our results suggest that the induction of PTEN-modulated apoptosis is one of the putative mechanisms of tumor suppressive activity by GLTSCR2.

Cell Death and Differentiation (2007) 14, 1872-1879; doi:10.1038/sj.cdd.4402204; published online 27 July 2007

Genetic alteration of chromosome 19 is a frequent event encountered in various human tumors, including diffuse gliomas. ${ }^{1,2}$ While the putative tumor suppressor gene localized to $19 \mathrm{q}$ has been narrowed to the $1.4 \mathrm{Mb}$ between the genetic markers $D 19 S 412$ and STD, it remains unidentified. $^{3-5}$ Glioma tumor suppressor critical region 2 (GLTSCR2/ protein interacting with the carboxyl terminus-1 (PICT-1)), initially identified as a herpes simplex infected cell proteins 22 (ICP22)- and infected cell proteins 0 (ICPO)-interacting protein, ${ }^{6}$ is one of the candidate genes localized in this critical region. Aside from its localization on the chromosome, several lines of evidence support the participation of GLTSCR2 in the suppression of tumor growth and development. GLTSCR2 physically interacts with phosphatase and tensin homolog deleted on chromosome 10 (PTEN) by phosphorylating the protein, ${ }^{7}$ which in turn regulates multiple cellular functions including proliferation, growth, differentiation, and apoptosis. While recent reports have shown that the knockdown of GLTSCR2 expression induces anchorage-independent tumor cell growth and decreases susceptibility to apoptosis, ${ }^{8}$ much remains unknown about the molecular mechanisms behind the tumor suppressive activity of GLTSCR2.

Tumor suppressor genes inhibit the development or growth of cancer cells via a diverse range of pathways such as cell cycle arrest and/or induction of apoptosis. For example, PTEN encodes a dual-specificity protein phosphatase with both substrate specificity and lipid phosphatase activity, 9,10 converting plasma membrane lipid phosphatidylinositol 3,4,5- trisphosphate $(\mathrm{PI}(3,4,5) \mathrm{P} 3)$ to $\mathrm{PI}(4,5) \mathrm{P} 2$, which antagonizes phosphoinositide 3-kinase (PI3K) and inactivates downstream protein kinases such as Akt and pyruvate dehydrogenase kinase isozyme 1 (PDK1). ${ }^{11-13}$ Consequently, the phosphatase activity of PTEN regulates multiple cellular functions including proliferation, growth, and apoptosis. However, it remains unclear whether the inactivation of survival genes, such as Akt, is sufficient for the induction of apoptosis by PTEN in cancer cells. Moreover, neither the molecular mechanisms nor the triggering factors of PTENinduced apoptosis have yet been clearly identified.

The purpose of this study was to investigate the molecular mechanisms of GLTSCR2 in cell death pathways in association with its binding partner PTEN. In this work, we show that besides phosphorylating PTEN, GLTSCR2 induces caspaseindependent apoptotic cell death modulated by PTEN. Our results suggest that the induction of PTEN-modulated cell death is one of the putative mechanisms of tumor suppressive activity by GLTSCR2.

\section{Results}

GLTSCR2 is a nucleus-localized protein. To examine the expression status of GLTSCR2, cell lysates of four glioblastoma cell lines (LN229, LN18, A172, and SNU1105) were subjected to western blotting. GLTSCR2 expression was conspicuously downregulated in SNU1105 cells as

\footnotetext{
${ }_{1}^{1}$ Department of Pathology, College of Medicine, Kyung Hee University, Seoul 130-701, Korea and ${ }^{2}$ Medical Research Center for Bioreaction to Reactive Oxygen Species, College of Medicine, Kyung Hee University, Seoul 130-701, Korea

*Corresponding author: J-H Park, Department of Pathology, College of Medicine, Kyung Hee University, \# 1 Hoegi-dong, Dongdaemoon-Koo, Seoul 130-701, Korea. Tel: + 822961 0533; Fax: + 822960 2871; E-mail: jhpark@khu.ac.kr Keywords: GLTSCR2; PTEN; cell death; glioma

Abbreviations: AIF, apoptosis-inducing factor; DAPI, 4'-6'-diamidino-2-phenylindole; GLTSCR2, glioma tumor suppressor candidate region 2; ICP0, infected cell proteins 0; ICP22, infected cell proteins 22; LDH, lactate dehydrogenase; NLS, nuclear localization signal; PDK1, pyruvate dehydrogenase kinase isozyme 1; PI3K, phosphoinositide 3-kinase; PICT-1, protein interacting with the carboxyl terminus-1; siRNA, small interfering RNA

Received 24.1.07; revised 07.6.07; accepted 20.6.07; Edited by B Zhivotovsky; published online 27.7.07
} 
compared with other glioblastoma cells (Figure 1a). Next, to ascertain the cellular localization of GLTSCR2, LN229 and SNU1105 cells were fractionated into their nuclear and cytosolic components and subjected to western blotting. As shown in Figure 1b, GLTSCR2 was detected in the nuclear compartment. Consistent with western blot data, expression with a GLTSCR2-expressing plasmid tagged with EGFP (pEGFP-GLT) localized to the nucleus in a discrete globular expression pattern (Figure 1c). These nuclear expression patterns were seen in all of the cell lines tested (data not shown). To map the putative nucleus localization sequence (NLS), we generated a series GLTSCR2 truncation mutants (Figure 2a). A sequential deletion of amino acids 1-346 did not change the globular nuclear localization pattern observed with wild-type GLTSCR2 (Figure 2b). In contrast, deletion mutants of the carboxy-terminus of GLTSCR2 (GLTA347479 and GLT $\Delta 181-479$ ) did not display the globular nuclear localization pattern observed with wild-type GLTSCR2; rather, they were dispersed throughout the nucleus (Figure 2b). Moreover, GLT/181-346 lost nuclear localization and was dispersed throughout the cytoplasm (Figure 2b). These results suggest that GLTSCR2 has two putative NLSs, one at either the amino- or carboxy termini, and that the carboxy-terminal NLS is crucial for the discrete globular localization pattern in the nucleus.

GLTSCR2 induces cell death with apoptotic features. To elucidate the functions of GLTSCR2 in cell growth and survival, we analyzed the effect of GLTSCR2 overexpression on cell viability. LN229 cells were transfected with empty pEGFP or pEGFP-GLT, and the cell viability was subsequently determined by the manual enumeration of dead cells with small, condensed and pyknotic or fragmented nuclei among the transfected cells at the indicated time intervals. As shown in Figure $3 a$, the number of dead cells was increased by $58 \%$ after $48 \mathrm{~h}$ of transfection. Morphologically, GLTSCR2-overexpressing cells displayed cytoplasmic condensation with a distorted cytoskeletal architecture and pyknotic nuclei with occasional fragmentation (Figure 3b, left panel). In addition, typical apoptotic changes, such as chromatin condensation and margination along the nuclear membrane, were noted with electron microscopy (Figure $3 b$, right panel). To further delineate the types of cell death induced by GLTSCR2, the percentage of cells with degraded DNA (hypodiploid DNA content) at the sub- $\mathrm{G}_{0} / \mathrm{G}_{1}$ fraction and phosphatidylserine externalization were determined. Flow cytometric analysis of Figure $3 c$ and d depict the increase of sub- $G_{0} / G_{1}$ fraction and early externalization of phosphatidylserine. Consistent with these data, levels of lactate dehydrogenase (LDH), which are suggestive of the plasma membrane rupture observed in necrotic cell death, were not significantly increased following $36 \mathrm{~h}$ of GLTSCR2 overexpression, a time point at which approximately $45 \%$ of GLTSCR2-overexpressing cells were dead (Figure $3 a$ and $e$ ). Our results suggest that overexpressed GLTSCR2 induced cell death with apoptotic features.

Nuclear localization is not required for the cell-killing activity of GLTSCR2. To gain insight into the molecular mechanisms of GLTSCR2-induced apoptosis, we transfected LN229 cells with each of the truncated mutant plasmid and determined the cell-killing potential of the mutants by a cell death assay in comparison with empty vector-transfected cells. As shown in the upper panel of Figure $4 \mathrm{a}$, the deletion of amino acids 181-346 (GLT $\Delta 1-346$
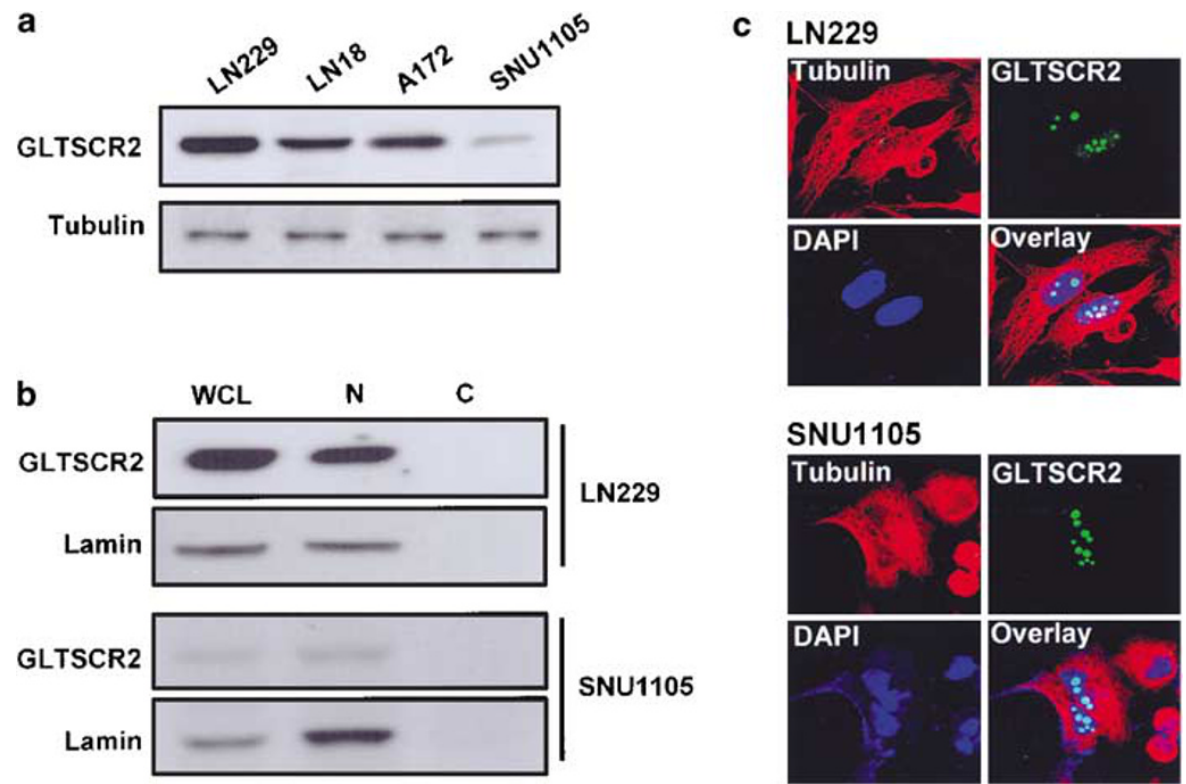

Figure 1 Expression and subcellular localization of GLTSCR2. (a) Western blots for GLTSCR2 expression in LN229, LN18, A172, and SNU1105 glioblastoma cell lines. (b) LN229 and SNU1105 cells were fractionated into their nuclear and cytosolic components and subjected to western blotting analysis using an anti-GLTSCR2 antibody. Cell fractionation was confirmed by western blot using an anti-lamin antibody. (c) LN229 and SNU1105 cells were transfected with $1 \mu \mathrm{g}$ of pEGFP-GLT plasmid. Following $18 \mathrm{~h}$ of transfection, subcellular localization was determined by immunocytochemistry with an anti-tubulin antibody and nuclear DAPI staining 
a

GLTSCR2
GLT $\Delta 1-65$
GLT $\Delta 1-149$
GLT $\Delta 1-346$
GLT $\Delta 347-479$
GLT $\Delta 181-479$
GLT/181-346

1
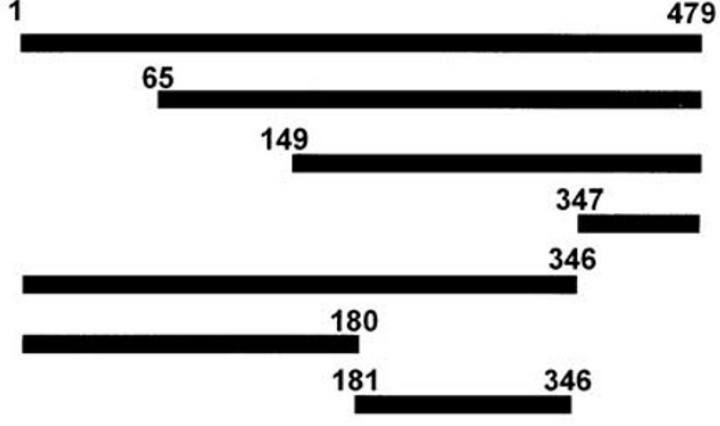

b

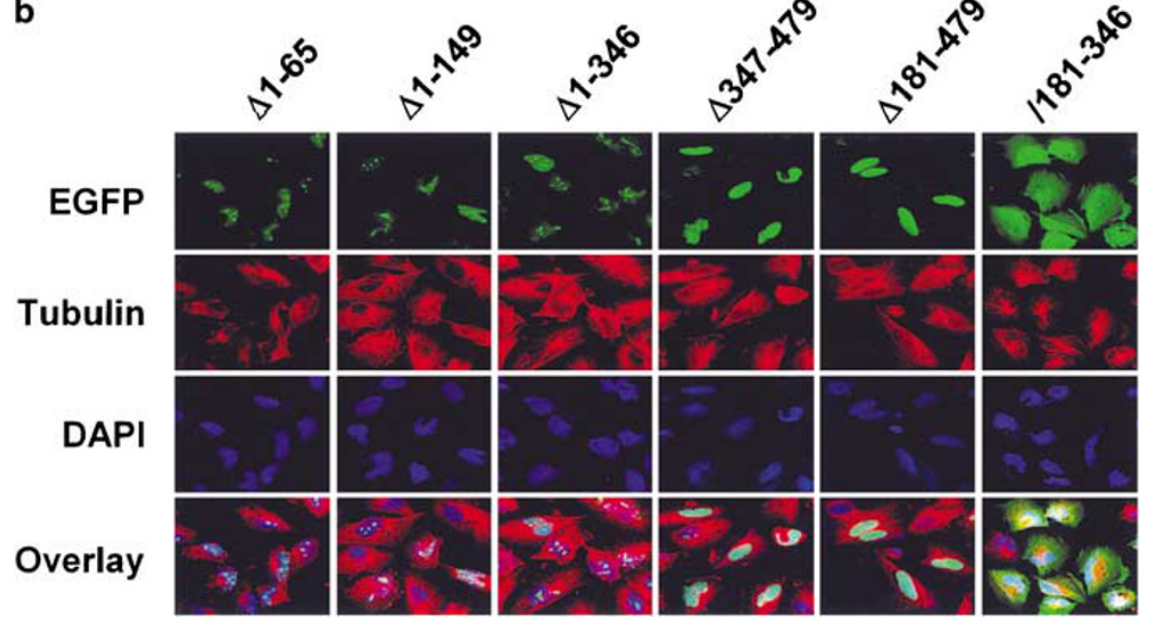

Figure 2 GLTSCR2 has two putative NLSs. (a) Schematic representation of GLTSCR2 truncation mutants; amino acids are numbered. (b) LN229 cells were transfected with $1 \mu \mathrm{g}$ of the indicated plasmid, which was tagged with EGFP, and the subcellular localization of the respective truncation mutant protein was determined by immunocytochemistry after staining with anti-tubulin antibody and DAPI

and GLT $\Delta 181-479$ ) resulted in a loss of cell-killing ability, suggesting that this domain is important for the cytotoxic effect of GLTSCR2. In addition, the nuclear localization of GLTSCR2 did not appear to be critical for the induction of cell death, as GLT/181-346, which was localized to the cytoplasm, retained the ability to kill cells (Figures $2 \mathrm{~b}$ and $4 \mathrm{a}$, upper panel). The mechanism of cell death by overexpression of the truncation mutant was basically the same as that of wild-type GLTSCR2 in that the subG $G_{0} / G_{1}$ fraction (Figure 4a, middle panel) and annexin-positive cells (Figure 4a, lower panel) were increased, and insignificant LDH release (data not shown) was noted. Equalization of mutant protein expression was confirmed by western blotting (Figure 4b). Collectively, our results suggest that when overexpressed, GLTSCR2 retains a pro-death property in both the nucleus and cytoplasm, and amino acids 181-346 are crucial for this cytotoxic effect.

Importance of PTEN in induction of cell death by GLTSCR2. Next, we wanted to know whether PTEN affected the cytotoxic potential of GLTSCR2. Glioblastoma cells with deleted PTEN (A172 ${ }^{14}$ and SNU1105) or wild-type PTEN (LN229 $9^{15}$ and $L N 18^{16}$ ) (Figure 5A) were transfected with pEGFP-GLT, and their pro-death effects were measured $48 \mathrm{~h}$ after transfection by a cell death assay and morphological assessment. Interestingly, cell death in PTEN-deleted cells was not significantly increased by
GLTSCR2 overexpression as compared with approximate $40 \%$ cell death in cells with PTEN expression (Figure 5B, lower panel). The difference in cell death for each cell line was not due to differences in GLTSCR2 expression levels, as the overexpression of GLTSCR2 levels by plasmid transfection were made similar for each cell line (Figure 5B, upper panel). Consistent with the results of the cell death assay, LN229 and LN18 cells displayed cytoplasmic shrinkage with condensed pyknotic nuclei, whereas A172 and SNU1105 cells did not show significant morphological changes (Figure 5C). To further elucidate the effects of PTEN on GLTSCR2-induced cell death, $\mathrm{A} 172^{\text {PTEN(+) }}$ and SNU1105 ${ }^{P T E N(+)}$, in which PTEN expression was restored by stably transfecting the PTEN expression plasmid pcDNAPTEN, were transfected with PEGFP-GLT, and alteration of cell death compared with the respective parent cell line was determined. As shown in Figure 5D, the cytotoxic effects of GLTSCR2 on A172 and SNU1105 were restored by ectopic PTEN expression. Equalization of transfected GLTSCR2 for parent and PTEN-stably transfected cells, as well as the restoration of PTEN expression, is shown in the upper panel of Figure 5D. Likewise, suppression of PTEN expression in LN229 and LN18 cells by small interfering RNA (siRNA) (siPTEN) decreased the sensitivity to GLTSCR2 overexpression (Figure 5E). The knockdown of PTEN by siPTEN and equalization of GLTSCR2 expression were confirmed by western blotting (Figure $5 \mathrm{E}$, upper panel). Our 

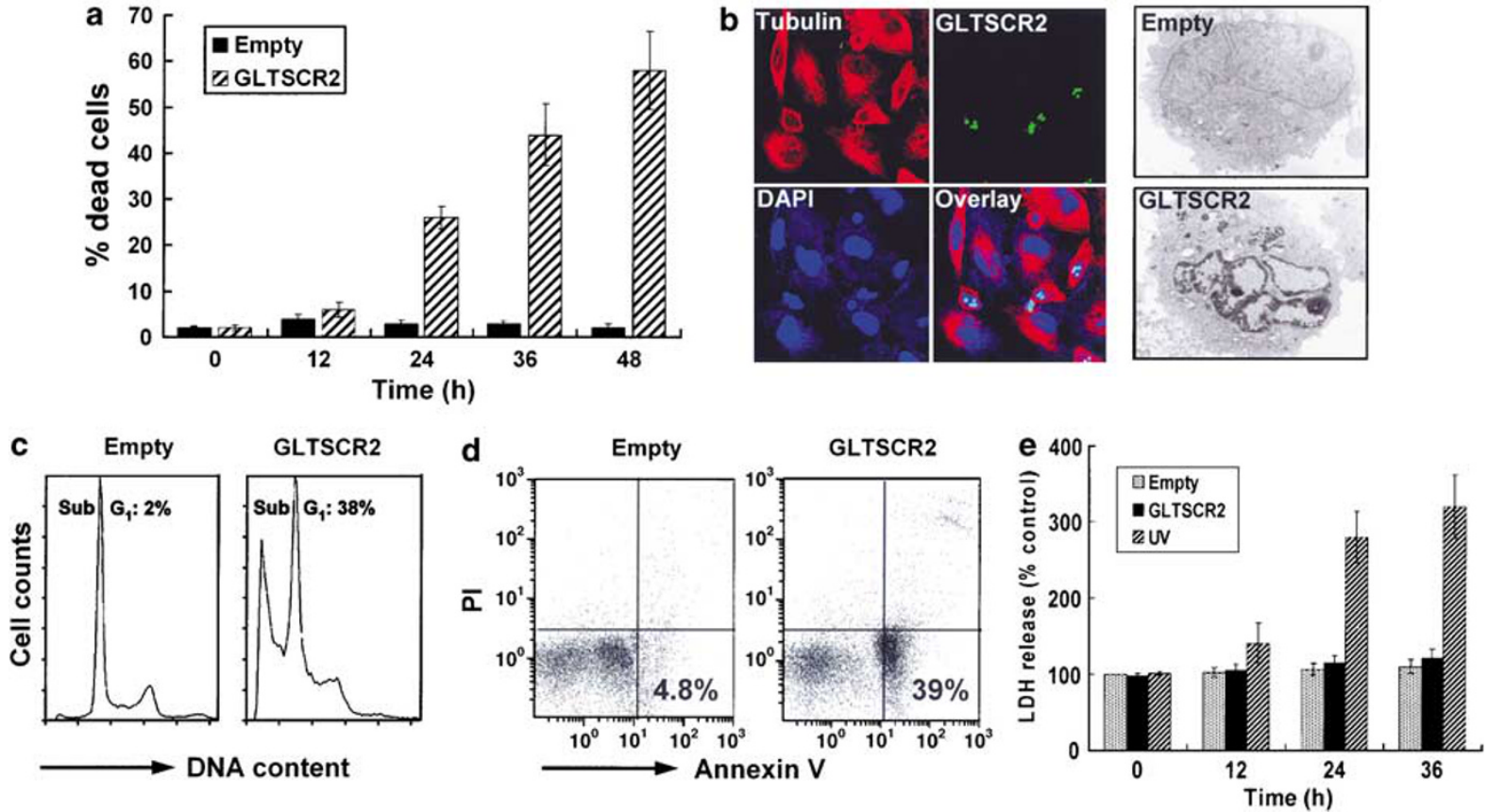

Figure 3 GLTSCR2 induces cell death with apoptotic features. (a) LN229 cells were transfected with $1 \mu \mathrm{g}$ of either empty or pEGFP-GLT vector. Dead cells were counted among transfected cells as described in the methods section at the indicated time intervals. (b) LN229 cells overexpressing GLTSCR2 (18 h after transfection) were viewed by confocal microscopy after staining with an anti-tubulin antibody and DAPI (left panel) or electron microscopy (right panel). (c) Cells transfected as in (a) were stained with propidium iodide (PI) and the sub- $G_{0} / G_{1}$ fraction was analyzed by flow cytometry. A representative histogram plot with the proportion of sub- $G_{0} / G_{1}$ fraction after $48 \mathrm{~h}$ of transfection is presented. (d) Following $12 \mathrm{~h}$ of transfection using empty or pEGFP-GLT vector, cells were double stained with Annexin V and Pl, and analyzed by flow cytometry. A representative plot is shown. (e) LDH released into culture medium was measured at the indicated time intervals after transfection. Cells irradiated with ultraviolet light (UV) were used as a positive control. Means \pm S.E.M. of three independent experiments are shown

data suggest that PTEN activity is required for the cytotoxic effects of GLTSCR2.

The cytotoxic activity GLTSCR2 is independent of its ability to phosphorylate PTEN. To further elucidate the relationship between the apoptotic activity of GLTSCR2 with PTEN phosphorylation, we transfected a series of GLTSCR2 truncation mutants into LN229 cells and determined the phosphorylation status of PTEN. Consistent with a previous report, ${ }^{7}$ overexpression of GLTSCR2 phosphorylated PTEN at Ser-380 (Figure 6). In addition, PTEN phosphorylation was not significantly affected by the deletion of the aminoterminal sequence (GLT $\Delta 1-65$, GLT $\Delta 1-149$, and GLT $\Delta 1-$ 346) (Figure 6). Interestingly, deletions of the GLTSCR2 carboxy-terminus (GLT $\Delta 347-479$, GLT $\Delta 181-479$, and GLT/ 181-346) resulted in a decrease of phosphorylation activity (Figure 6), suggesting that the carboxy-terminal domain of GLTSCR2 (amino acids 347-479) is responsible for the phosphorylation of PTEN. Taken with the results shown above, our data suggest that GLTSCR2 exerts its cytotoxic activity independent of PTEN phosphorylation.

Divergence between GLTSCR2- and PTEN-induced apoptotic pathways. It has been well documented that PTEN induces apoptotic cell death via many diverse signaling pathways such as survivin ${ }^{17}$ or $\mathrm{p} 53 .{ }^{18} \mathrm{We}$ therefore sought to determine whether GLTSCR2 activated PTEN-mediated apoptotic mechanisms or facilitated cell death via divergent pathways independent of PTEN. LN229 cells were transfected with pEGFP-GLT or pEGFP-PTEN, and a caspase- 3 substrate cleavage assay was performed at the indicated time points. Figure 7 a shows that caspase- 3 was not activated during GLTSCR2-induced cell death, while PTEN and a positive Bax control were able to induce activation of caspase-3. In addition, a pan-caspase inhibitor (z-VAD) did not suppress cell death induced by GLTSCR2 whereas it did suppress PTEN-induced cell death (Figure $7 \mathrm{~b}$ ). In further support of caspase independence in GLTSCR2-induced cell death pathways, we were unable to detect the release of cytochrome $c$ from mitochondria (Figure $7 \mathrm{c}$ ) or the dissipation of the mitochondrial membrane potential (data not shown). Altogether, these results suggest that GLTSCR2 facilitates cell death through a caspase- and mitochondria-independent pathway, divergent from PTEN-mediated pathways.

\section{Discussion}

GLTSCR2 is a putative tumor suppressor gene localized on chromosome $19 \mathrm{q}$ and is frequently altered in human brain tumors, including diffuse gliomas. ${ }^{1,2}$ An accumulating body of evidence clearly shows that GLTSCR2 retains the tumor suppressive activities, such as phosphorylation and stabilization of PTEN, as well as sensitization to apoptosis; ${ }^{7,8}$ however, much remains unknown about the molecular mechanisms and pathways behind the activity of GLTSCR2. 

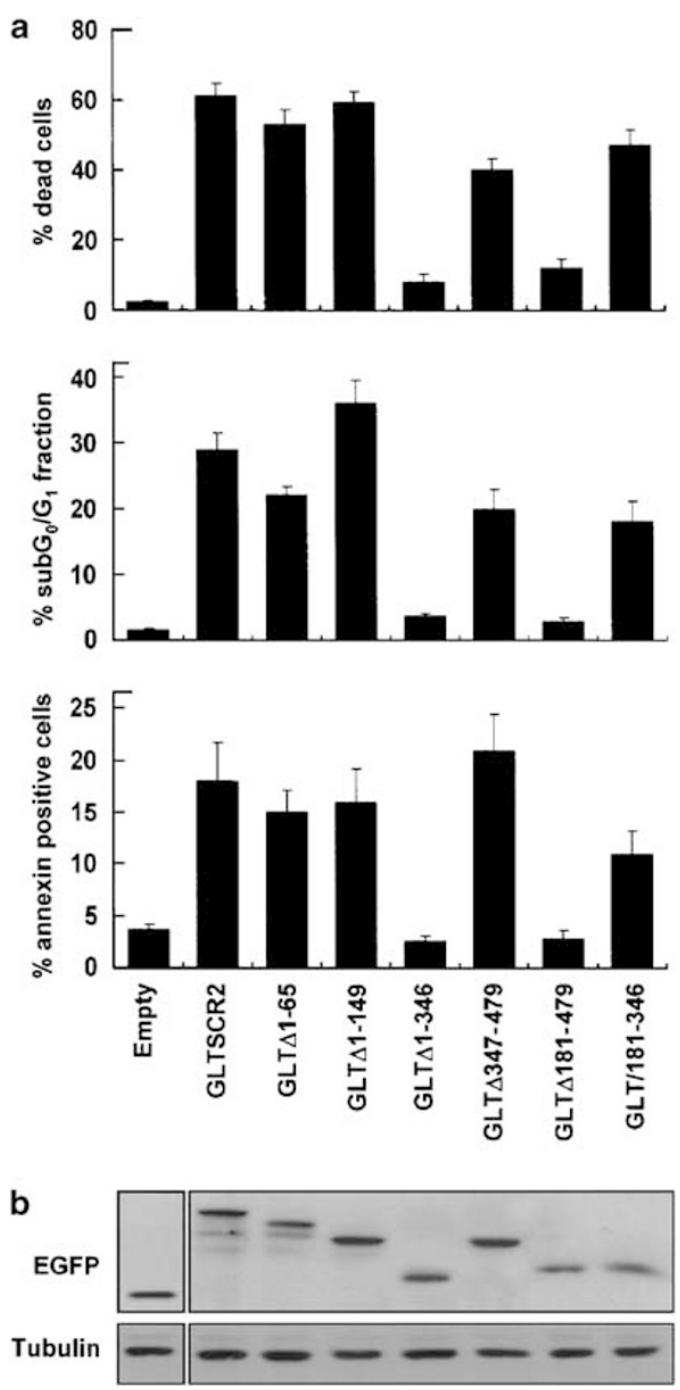

Figure 4 The cytotoxic domain of GLTSCR2. (a) LN229 cells were transfected with $1 \mu \mathrm{g}$ of the empty, GLTSCR2, or the indicated mutant plasmid, and the cell death assay (upper panel) and flow cytometric analysis of sub- $G_{0} / G_{1}$ fraction (middle panel) and annexin-positive cells (lower panel) were performed as in Figure 3. (b) Equalization of each transfected protein was confirmed by western blotting using an anti-EGFP antibody (upper panel). Loading controls using an anti-tubulin antibody are shown in the lower panel

We performed this study to investigate the biological function of GLTSCR2 and its mechanisms of tumor suppression.

We first looked at the expression and subcellular localization of GLTSCR2 and found that it was expressed as multiple small discrete globular structures within the nucleus. Truncation mutation analysis revealed the presence of two NLS at both the amino- and carboxy-terminals. Consistent with this finding, computer-assisted NLS analysis (http://cubic.bioc. columbia.edu/) indicated the presence of two NLSs, 34-39 (LRRRRR) and 387-392 (RRRRRR), at the amino- and carboxy-terminus, respectively. However, the two NLSs of GLTSCR2 appeared to have different functions, whereby mutant GLTSCR2, which has a deletion of the carboxyterminal sequence (GLT $\Delta 347-479$ ), lost the globular localization pattern and was dispersed throughout the nucleoplasm, while the amino-terminal deletion (GLT $\Delta 1-65)$ retained multiple, small discrete patterns as did the wild-type GLTSCR2. These data suggest that the carboxy-terminal sequence of GLTSCR2 may interact with a target protein within the nucleus. In support of our data, the carboxy-terminal 130 amino acids have been defined as the binding domain for ICPO of the herpes virus. ${ }^{6}$ No partner molecules of GLTSCR2 within the nucleus have been identified, although a yeast twohybrid screen to detect any binding proteins is currently being developed in our laboratory. In contrast to the carboxyterminal sequence, the amino-terminal sequence was sufficient for the translocation of GLTSCR2 to the nucleus.

We next analyzed the cytotoxic activity of GLTSCR2 to evaluate its potential for tumor suppression. Morphological and biochemical parameters, including fluorescent and electron microscopy, DNA ploidy, and annexin V staining, categorized GLTSCR2 as a member of a group that induces cell death with apoptotic features; however, GLTSCR2 appeared to be different from other proapoptotic proteins. While large parts of pro-death proteins require targeting to a specific organelle for their cytotoxic activity, some execute their functions irrespective of intracellular localization. ${ }^{19,20}$ In the case of GLTSCR2, its cytotoxic potentials were retained irrespective of its localization, both in the cytoplasm and nucleus, as GLTSCR2/181-346, which lacked any NLS, retained killing activity. The presence of the death-inducing domain consisting of amino acids 181-346 was important for cytotoxic activity rather than subcellular localization, because the deletion of amino acids 1-347 or 181-479 markedly reduced the cytotoxic potential of GLTSCR2. It remains to be determined whether this region participates in protein-protein interactions with intracellular death-inhibitory or apoptoticinducing target proteins.

The phosphatase activity of PTEN, which antagonizes the PI3 kinase and thus dephosphorylates downstream protein kinase Akt and PDK1, modulates a diverse range of signaling cascades including cell growth, survival, or death. ${ }^{12,13}$ Our data obtained from the truncation mutants revealed that the amino-terminus of GLTSCR2 participates in the phosphorylation of PTEN, whereas amino acids 181-346, which lack the phosphorylation domain for PTEN, are responsible for the cytotoxic activity of GLTSCR2. The apparent separation of the PTEN phosphorylation domain from the cytotoxic domain suggests that GLTSCR2 facilitates cell death independent of the PTEN-Akt signaling cascades. In support of this assumption, the cell death pathway of GLTSCR2 is completely different from that of PTEN. Data obtained from our caspase assay, cell fractionation assay, and determination of mitochondrial parameters showed that GLTSCR2 induced caspase-independent cell death without mitochondrial perturbation or mitochondrial release of death proteins. These findings contradict established PTEN-induced cell death mechanisms, as PTEN regulates cell death via a caspaseand mitochondria-dependent pathway. ${ }^{21}$ Nevertheless, insensitivity of PTEN-deleted A172 and SNU444 cells to GLTSCR2 overexpression and restoration of sensitivity to GLTSCR2 by ectopic PTEN expression indicated that normal PTEN activity was required for cell death induced by GLTSCR2. Further studies demonstrating the molecular triggering mechanisms for GLTSCR2 cytotoxicity by PTEN 
A

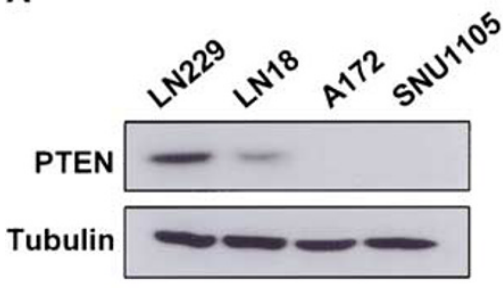

C LN229

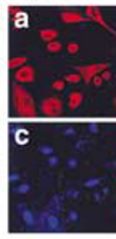

A172

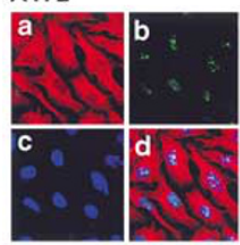

LN18

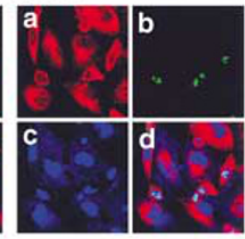

SNU1105

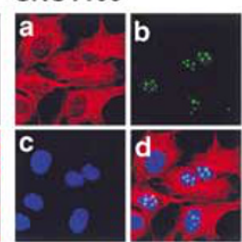

B$$
\text { }
$$

60
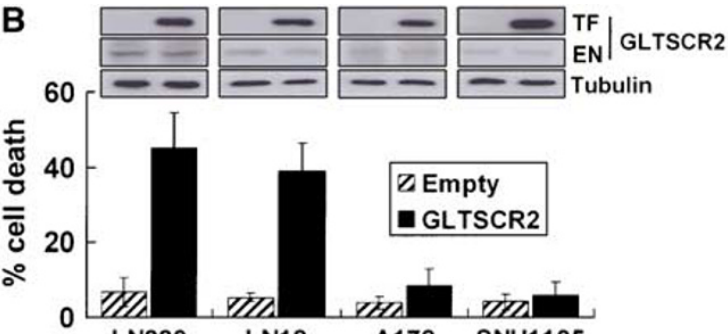

I

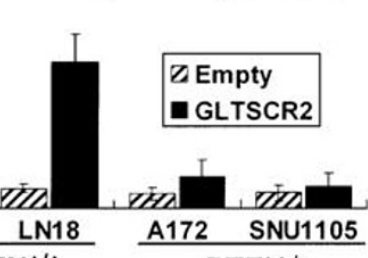

PTEN $^{+/+}$ PTEN ${ }^{1 /}$

D

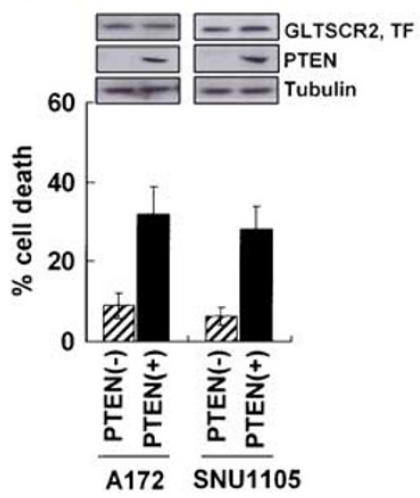

E

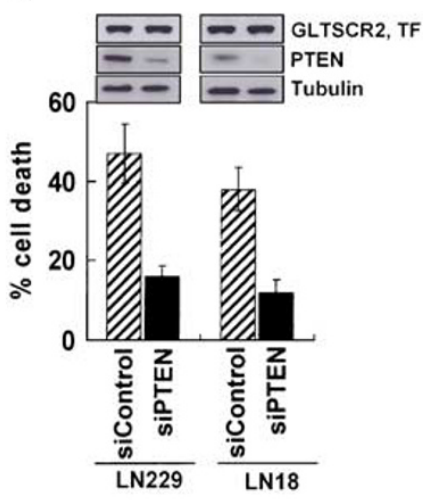

Figure 5 PTEN is crucial for cell death induced by GLTSCR2. (A) Total cell lysates from the indicated cell lines were subjected to western blot for PTEN expression. Tubulin was used as control for loading. (B) The indicated cells were transfected with $1 \mu \mathrm{g}$ of an empty vector control or GLTSCR2 expression plasmid, and dead cells were subsequently counted among the empty- or GLTSCR2-transfected cells (lower panel). Expression levels of transfected (TF) and endogenous (EN) GLTSCR2 are shown (upper panel). (C) Morphological assessment of (b) after tubulin and DAPI staining. a, tubulin; b, EGFP; c, DAPI; d, overlay. (D) Parents (PTEN(-)) and PTEN-stably transfected (PTEN $(+))$ cells were transfected with $1 \mu \mathrm{g}$ of pEGFP-GLT, and dead cells were counted among GLTSCR2-overexpressing cells $36 \mathrm{~h}$ after of transfection. Expression levels of transfected GLTSCR2 and PTEN with loading control are shown in the upper panel. (E) Indicated cells were transfected with siControl or siPTEN for $48 \mathrm{~h}$ and then transfected with $1 \mu \mathrm{g}$ of pEGFP-GLT. The cell death assay was performed $36 \mathrm{~h}$ after pEGFP-GLT transfection. Expression levels of transfected GLTSCR2 and PTEN with loading control are shown in the upper panel. Means \pm S.E.M. of three independent experiments are shown

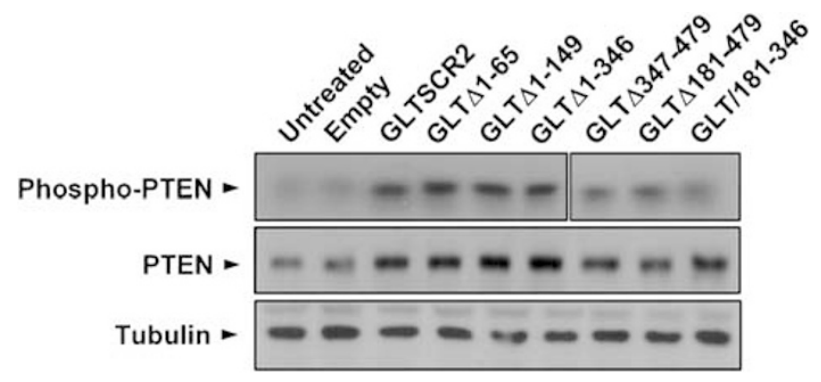

Figure 6 The carboxy-terminus of GLTSCR2 is crucial for PTEN phosphorylation. LN229 cells were left untreated or transfected with either an empty vector or the indicated plasmid and subjected to western blotting for Ser380-phosphorylated and total PTEN $18 \mathrm{~h}$ after transfection

will be needed in order to understand the tumor suppressive function of GLTSCR2.

In summary, we showed that GLTSCR2 not only affected PTEN activity by phosphorylation but also triggered caspaseand mitochondria-independent cell death mechanisms modulated by PTEN. These results suggest that the tumor suppressive function of GLTSCR2 and PTEN are tightly interconnected.

\section{Materials and Methods}

Antibodies and reagents. Anti-GLTSCR2 polyclonal antibody was produced by immunizing a rabbit with keyhole limpet hemocyanin-conjugated amino-acid residues 178-193 (CTRAKPGPQDTVERPF) of human GLTSCR2; antibody was purified from the immune serum by affinity chromatography. Anti-PTEN, -phosphoPTEN, and -cytochrome $c$ antibodies were purchased from Abcam (Cambridge, UK). Anti-lamin antibody was purchased from Santa Cruz Biotechnology, Inc. (Santa Cruz, CA, USA). Unless otherwise specified, all other reagents were obtained from Sigma-Aldrich, Inc. (St Louis, MO, USA).

Cell culture, cell viability, and cell death assay. The human LN229, LN18, and A172 glioblastoma cell lines were obtained from the American Type Culture Collection (Rockville, MD, USA). The SNU1105 cell line was purchased from the Korean Bank of Cancer Cells (Seoul, Korea). All cells were cultured in RPMI 1640 medium (Gibco BRL, CA, USA) containing 10\% fetal bovine serum under a $5 \% \mathrm{CO}_{2}$ atmosphere at $37^{\circ} \mathrm{C}$. Cells stably expressing PTEN were generated by transfecting $\mathrm{A} 172$ and SNU1105 cells with a PTEN-expressing plasmid (pcDNA-PTEN) and selection with G418. The cell death assay was performed as follows: dead cells exhibiting altered nuclear morphology, such as fragmentation or a condensed pyknotic chromatin pattern among transfected cells, were enumerated manually under a fluorescence microscope following $4^{\prime}, 6^{\prime}$ diamidino-2-phenylindole (DAPI) staining. For quantitative determination of the sub$\mathrm{G}_{0} / \mathrm{G}_{1}$ fraction, cells were stained with propidium iodide $(\mathrm{PI})$ and analyzed using flow cytometry ( $\mathrm{FC} 500$, Beckerman-Coulter) $48 \mathrm{~h}$ after transfection. Annexin $\mathrm{V}$ staining was performed according to the manufacturer's instructions (Calbiochem, CA, USA) $12 \mathrm{~h}$ after transfection. Lactate dehydrogenase (LDH) released into culture medium was measured using an LDH assay kit (Calbiochem). 

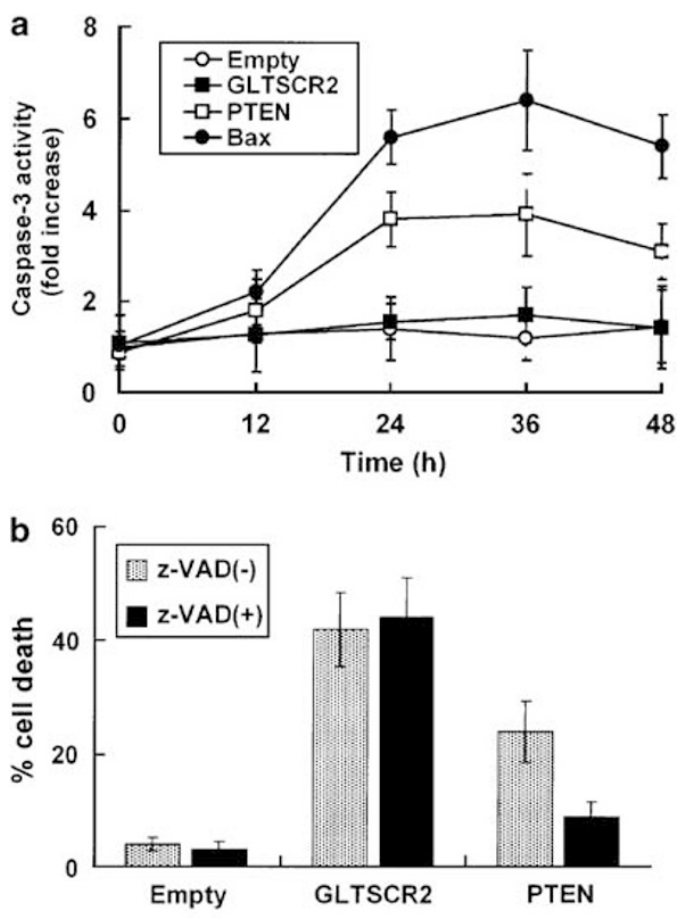

C

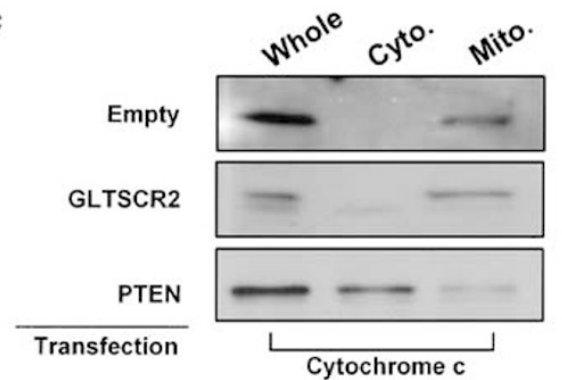

Figure 7 GLTSCR2 induces caspase-independent cell death without involving mitochondrial apoptotic cascades. (a) LN229 cells were transfected with empty, GLTSCR2, PTEN, or Bax expression plasmid, and caspase-3 activity was determined at the indicated time intervals; Bax was used as a positive control. (b) Cells were transfected with $1 \mu \mathrm{g}$ of empty, GLTSCR2, or PTEN expression plasmid in the presence or absence of $20 \mu \mathrm{M} \mathrm{z}$-VAD treated $3 \mathrm{~h}$ prior to transfection. Dead cells were counted among cells with overexpression of GLTSCR2, $36 \mathrm{~h}$ after transfection. Data represent the mean \pm S.D. of three independent experiments. (c) Cells were transfected with empty, pEGFP-GLT, or pEGFP-PTEN, fractionated by differential centrifugation, and then subjected to western blotting for cytochrome $c$ release. Whole, whole cell lysates; Cyto., cytosolic fraction; Mito., mitochondrial fraction

Plasmid construction and transient transfection. DNAs encoding full-length GLTSCR2 (Accession no. NM_015710) and Bax (Accession no. L22473) were amplified by polymerase chain reaction (PCR) from a human kidney CDNA library and cloned into pcDNA3.1/V5 (Invitrogen Corp., CA, USA) or pEGFP (Clontech Laboratories, Inc., CA, USA) vectors. Various deletion mutants of GLTSCR2 were constructed by PCR and subcloned into the pcDNA3.1/V5 or pEGFP vectors. Glioblastoma cells $\left(3 \times 10^{5} / 10 \mathrm{~cm}\right.$ culture dish) were transiently transfected with each plasmid using Magnetofection according to the manufacturer's protocol (Chemicell, Berlin, Germany).

Determination of mitochondrial membrane potential. Transfected glioblastoma cells were cultured under the indicated conditions, and Mitotracker Red CMXRos (Molecular Probes, Inc.) was added to the medium at a final concentration of $400 \mathrm{nM}$. After $20 \mathrm{~min}$ of incubation, the cells were harvested and washed three times with phosphate-buffered saline (PBS). The fluorescence intensity was measured by flow cytometry (FC 500, Beckmann-Coulter).

Cell fractionation and western blotting. Cell fractionation was performed as previously described. ${ }^{22}$ Extracts from total cells or cell fractions were suspended in lysis buffer $(10 \mathrm{mM}$ Tris- $\mathrm{HCl}(\mathrm{pH} 7.4), 1 \%$ Triton X-100, $0.1 \%$ sodium deoxycholate, $0.1 \%$ SDS, $150 \mathrm{mM} \mathrm{NaCl}, 1 \mathrm{mM}$ EDTA, $1 \mathrm{mM}$ EGTA, $0.5 \mathrm{mM}$ phenylmethylsulfonyl fluoride, $1.1 \mathrm{mM} \mathrm{Na}_{3} \mathrm{VO}_{4}$, and $10 \mathrm{mM} \mathrm{NaF}$ ). Extracted proteins were separated by SDS-PAGE on $12 \%$ polyacrylamide gels and electrophoretically transferred onto nylon membranes. The membranes were probed with primary antibody, followed by incubation with horseradish peroxidase-coupled secondary antibody. Detection was performed with a chemiluminescence-based detection kit (Amersham Pharmacia Biotech).

Caspase assays. Caspase- 3 activities were measured with the colorimetric caspase-3 assay kit (Calbiochem) according to the manufacturer's instructions.

Immunocytochemistry and electron microscopy. Glioblastoma cells were fixed with $4 \%$ paraformaldehyde in PBS and permeabilized with $0.1 \%$ Triton X100. After blocking with $3 \%$ bovine serum albumin in PBS, the cells were incubated with anti-cytochrome $c$, anti-apoptosis-inducing factor (AIF), anti-smac/DIABLO, anti-HtrA2/Omi, or anti-endonuclease $\mathrm{G}$, and anti-tubulin antibody for $1.5 \mathrm{~h}$ at room temperature. Afterwards, the cells were stained with secondary antibody conjugated with fluorescein isothiocyanate or Texas Red and viewed after DAPI staining under a confocal microscope (META 510, Zeiss). For ultrastructural studies, cells were fixed with $2.5 \%$ glutaldehyde in $0.1 \mathrm{M}$ phosphate buffer, $\mathrm{pH} 7.3$, treated with osmium tetroxide in $0.1 \mathrm{M}$ phosphate buffer, and then embedded in Epon (Poly/Bed $812^{\circledR}$ embedding media/DMP-30 kit, PolyScience, Germany). The cells were then observed under an electron microscope (H7100, Hitachi, Japan).

Suppression of endogenous GLTSCR2 expression by siRNA. Pairs of 19-nucleotide sense and antisense RNA oligomers were chemically synthesized independently and annealed by Bioneer Corp. (Daejeon Korea). The oligonucleotides for GLTSCR2 were as follows: sense, 5'-GCA CAG GCU GCG GGU ACA G-3', and antisense, 5'-CUG UAC CCG CAG CCU GUG C-3', which corresponded to human GLTSCR2 coding nucleotides 1455-1475. The selected sequence (siA11) was subjected to a BLAST search to ensure that no other part of the human genome sequence other than GLTSCR2 was targeted. The introduction of siRNA was carried out using Lipofectamine 2000 (Invitrogen) according to the manufacturer's instructions.

Acknowledgements. This work was supported by the Korea Science \& Engineering Foundation through the Medical Research Center for Bioreaction to Reactive Oxygen Species at Kyung Hee University (no. R13-2002-020-01002-0) and the Neurobiology Research Program.

1. Smith JS, Tachibana I, Pohl U, Lee HK, Thanarajasingam U, Portier BP et al. A transcript map of the chromosome 19q-arm glioma tumor suppressor region. Genomics 2000; 64: 44-50.

2. Trent JM, Kaneko Y, Mitelman F. Report of the committee on structural chromosome changes in neoplasia. Cytogenet Cell Genet 1989; 51: 533-562.

3. Rosenberg JE, Lisle DK, Burwick JA, Ueki K, von Deimling A, Mohrenweiser HW et al. Refined deletion mapping of the chromosome 19q glioma tumor suppressor gene to the D19S412-STD interval. Oncogene 1996; 13: 2483-2485.

4. Ritland SR, Ganju V, Jenkins RB. Region-specific loss of heterozygosity on chromosome 19 is related to the morphologic type of human glioma. Genes Chromosomes Cancer 1995; 12: 277-282

5. Rubio MP, Correa KM, Ueki K, Mohrenweiser HW, Gusella JF, von Deimling A et al. The putative glioma tumor suppressor gene on chromosome 19q maps between APOC2 and HRC. Cancer Res 1994; 54: 4760-4763.

6. Bruni R, Fineschi B, Ogle WO, Roizman B. A novel cellular protein, p60, interacting with both herpes simplex virus 1 regulatory proteins ICP22 and ICPO is modified in a cell-typespecific manner and is recruited to the nucleus after infection. J Virol 1999; 73: 3810-3817.

7. Okahara F, Ikawa H, Kanaho $\mathrm{Y}$, Maehama T. Regulation of PTEN phosphorylation and stability by a tumor suppressor candidate protein. J Biol Chem 2004; 279: 45300-45303.

8. Okahara F, Itoh K, Nakagawara A, Murakami M, Kanaho Y, Maehama T. Critical role of PICT-1, a tumor suppressor candidate, in phosphatidylinositol 3,4,5-trisphosphate signals and tumorigenic transformation. Mol Biol Cell 2006; 17: 4888-4895. 
9. Myers MP, Stolarov JP, Eng C, Li J, Wang SI, Wigler MH et al. P-TEN, the tumor suppressor from human chromosome 10q23, is a dual-specificity phosphatase. Proc Nat Acad Sci USA 1997; 94: 9052-9057.

10. Gu J, Tamura M, Yamada KM. Tumor suppressor PTEN inhibits integrin- and growth factor-mediated mitogen-activated protein (MAP) kinase signaling pathways. J Cell Bio 1998; 143: 1375-1383.

11. Maehama T, Dixon JE. The tumor suppressor, PTEN/MMAC1, dephosphorylates the lipid second messenger, phosphatidylinositol 3,4,5-trisphosphate. J Biol Chem 1998; 273 13375-13378.

12. Leslie NR, Downes CP. PTEN: the down side of PI 3-kinase signalling. Cell Signal 2003 14: $285-295$.

13. Downward J. PI 3-kinase, Akt and cell survival. Semin Cell Dev Biol 2004; 15 177-182.

14. Adachi J, Ohbayashi K, Suzuki T, Sasaki T. Cell cycle arrest and astrocytic differentiation resulting from PTEN expression in glioma cells. J Neurosurg 1999; 91 822-830.

15. Maier D, Jones G, Li X, Schonthal AH, Gratzl O, Van Meir EG et al. The PTEN lipid phosphatase domain is not required to inhibit invasion of glioma cells. Cancer Res 1999; 59: 5479-5482
16. Park MJ, Kim MS, Park IC, Kang HS, Yoo H, Park SH et al. PTEN suppresses hyaluronic acid-induced matrix metalloproteinase-9 expression in U87MG glioblastoma cells through focal adhesion kinase dephosphorylation. Cancer Res 2002; 62: 6318-6322.

17. Wu ZX, Song TB, Li DM, Zhang XT, Wu XL. Overexpression of PTEN suppresses growth and induces apoptosis by inhibiting the expression of survivin in bladder cancer cells. Tumour Biol 2006; 28: 9-15.

18. Zhou M, Gu L, Findley HW, Jiang R, Woods WG. PTEN reverses MDM2-mediated chemotherapy resistance by interacting with p53 in acute lymphoblastic leukemia cells. Cancer Res 2003; 63: 6357-6362.

19. Wolter KG, Hsu YT, Smith CL, Nechushtan A, Xi XG, Youle RJ. Movement of Bax from the cytosol to mitochondria during apoptosis. J Cell Biol 1997; 139: 1281-1292.

20. Ray R, Chen G, Vande Velde C, Cizeau J, Park JH, Reed JC et al. BNIP3 heterodimerizes with $\mathrm{Bcl}-2 / \mathrm{Bcl}-\mathrm{X}(\mathrm{L})$ and induces cell death independent of a Bcl-2 homology $3(\mathrm{BH} 3)$ domain at both mitochondrial and nonmitochondrial sites. J Biol Chem 2000; 275: 14391448

21. Zhu Y, Hoell P, Ahlemeyer B, Krieglstein J. PTEN: a crucial mediator of mitochondriadependent apoptosis. Apoptosis 2006; 11: 197-207.

22. Kim J, Ahn H, Ryu J, Suk K, Park J. BH3-only protein Noxa is a mediator of hypoxic cell death induced by hypoxia-inducible factor 1alpha. J Exp Med 2004; 199: 113-124.

Supplementary Information accompanies the paper on Cell Death and Differentiation website (http://www.nature.com/cdd) 